\title{
Psychological homeostasis
}

David F Marks

Potential competing interests: The author(s) declared that no potential competing interests exist.

Psychological homeostasis is a fundamental process enabling the satisfaction of psychological needs. Failure to meet psychological needs may lead to harm, illness or death. 